\title{
NeuRad detector prototype pulse shape study
}

I. Muzalevsky ${ }^{1,3,4, \star}$, V. Chudoba ${ }^{1,3, \star \star}$, S. Belogurov ${ }^{1}$, O. Kiselev ${ }^{5}$, A. Bezbakh ${ }^{1,3}$, A. Fomichev $^{1}$, S. Krupko ${ }^{1}$, R. Slepnev ${ }^{1}$, D. Kostyleva ${ }^{5}$, A. Gorshkov ${ }^{1}$, E. Ovcharenko², and V. Schetinin ${ }^{2}$ for the EXPERT/Super-FRS Experiment Collaboration

${ }^{1}$ Flerov Laboratory of Nuclear Reactions, JINR, 141980 Dubna, Russia;

${ }^{2}$ Laboratory of Information Technologies, JINR, 141980 Dubna, Russia;

${ }^{3}$ Institute of Physics, Silesian University in Opava, 74601 Opava, Czech Republic;

${ }^{4}$ Dubna State University, 141982 Dubna, Russia;

${ }^{5}$ GSI Helmholtzzentrum für Schwerionenforschung, 64291 Darmstadt, Germany

\begin{abstract}
The EXPERT setup located at the Super-FRS facility, the part of the FAIR complex in Darmstadt, Germany, is intended for investigation of properties of light exotic nuclei. One of its modules, the high granularity neutron detector NeuRad assembled from a large number of the scintillating fiber is intended for registration of neutrons emitted by investigated nuclei in low-energy decays. Feasibility of the detector strongly depends on its timing properties defined by the spatial distribution of ionization, light propagation inside the fibers, light emission kinetics and transition time jitter in the multi-anode photomultiplier tube. The first attempt of understanding the pulse formation in the prototype of the NeuRad detector by comparing experimental results and Monte Carlo (MC) simulations is reported in this paper.
\end{abstract}

\section{Introduction}

Properties of exotic nuclei represent one of the most important fields in modern nuclear physics. Such uncommon nuclei are characterized by a large excess of neutrons or protons and are located far from the nuclear stability. As far as the binding energy decreases, one may observe the transition from the discrete spectra to the nuclear resonances with many overlapping states and, as a consequence, such unique phenomena as neutron halo, soft mode of dipole excitation and others could be observed. Moreover, new decay channels, including many-body, are becoming open [1].

One of the best facilities for production of beams of unstable exotic nuclei will be the Super-FRS fragment separator at FAIR (Facility for Antiproton and Ion Research) [2]. The project EXPERT (EXotic Particle Emission and Radioactivity by Tracking [3]) dedicated to study of properties of exotic nuclei is a part of scientific program of the Super-FRS Experiment Collaboration. The EXPERT setup will be situated in intermediate focal planes of the Super-FRS beam line. The setup consists of six modules [4]: (i) The secondary target for

\footnotetext{
$\star$ e-mail: muzalevsky@jinr.ru

$\star \star$ e-mail: chudoba@jinr.ru
} 
production of the nuclei of interest (ii) The radiation-hard silicon strip detector for Timeof-Flight measurements and triggering. (iii) The microstrip silicon $(\mu \mathrm{Si})$ tracking detectors. (iv) The gamma-ray and light particles detector system GADAST (GAmma-ray Detectors Around Secondary Target). (v) The OTPC (Optical Time Projection Chamber) for radioactivity studies by the implantation-decay method. (vi) The NeuRad (Neutron Radioactivity) fine-resolution neutron detector.

Moreover, the EXPERTroot framework [5] for simulations of the experiments and processing of the experimental data and the software for simulation of theoretical distributions are indissociable parts of the project.

The neutron detector NeuRad is aimed at providing precise information on angular correlations between the neutrons and the heavy fragment emitted in the decay of the nucleus of interest. The heavy fragment will be identified by Super-FRS beam diagnostic elements situated downstream of the secondary target, whereas trajectories of a heavy fragment and neutrons will be reconstructed by an array of the $\mu \mathrm{Si}$ detectors and the NeuRad detector respectively. This information will be used to determine the decay energy of the investigated nucleus and its lifetime.

The NeuRad will be constructed from a large number of scintillating fibers ( $\approx 10000$ units). Each fiber will have a square cross section of $3 \times 3 \mathrm{~mm}^{2}$ and a length of $1 \mathrm{~m}$. Scintillating fibers will be grouped into bundles fitting the photosensors e.g. multi-anode photomultiplier tubes (MAPMT). The MAPMTs will be mounted to the bundles so that the area of the each pixel will completely overlap the front surface of one fiber. The NeuRad will be placed $28 \mathrm{~m}$ downstream of the secondary target in such a way that the fibers will be oriented along the beam direction [6]. It will provide sufficient detection efficiency and high angular resolution for the neutrons with energies $200-800 \mathrm{MeV}$ in the lab frame.

The neutrons penetrating the fibers will undergo different interaction processes. The most important one for the neutron registration is the elastic neutron-proton scattering. In such a case the recoil proton will induce scintillation light inside the fiber. The light emitted within the full reflection angle travels to the MAPMTs located at the both ends of the fiber. With such configuration the total angular acceptance of the detector will be about $12 \mathrm{mrad}$. Such a value is sufficient for registration of the neutrons with low transversal momenta, as expected if the energy release in the decay is in the range of $0.1-100 \mathrm{keV}$.

In the case of a single scattering of the neutron inside the NeuRad and sufficiently high energy release in one fiber, the position of the fired fiber gives information about the transversal coordinates of the interaction point. The time stamps of the signals from both the MAPMTs allow to derive the longitudinal position of the hit (spatially restricted energy deposit) providing a correction of the time-of-flight measurement. The time-of-flight information is used for the determination of the neutron kinetic energy.

If several fibers are fired due to the neutron re-scattering in detector volume or sharing of the energy deposited by the recoil proton, the first interaction point may be reconstructed by searching for the earliest signal in the upstream MAPMT. Besides, timing information may be used in more sophisticated analysis of the events with multiple hits.

Feasibility of the detector strongly depends on its timing properties. Each detector pulse is a superposition of several single photoelectron pulses whose distribution in time and amplitude is defined by the spatial distribution of ionization, light propagation inside the fibers, light emission kinetics, transition time jitter in the MAPMT, pulse shape and amplitude spectrum of the single photoelectron.

The pulse shapes produced by the neutrons hitting the exactly known interaction point are difficult to study experimentally. However MC study of the efficiency and precision of the 
neutron detector is possible provided that the MC is validated in more simple measurements. The first attempt of understanding the pulse formation in the prototype of the NeuRad detector by comparing experimental results and MC simulations is reported in this paper.

\section{Test of the NeuRad prototype}

The first investigation of the NeuRad detector was performed recently in the Flerov Laboratory of Nuclear Reactions JINR, Dubna. The NeuRad prototype consisted of one bundle of 256 BCF-12 [7] scintillating fibers having a square cross section of $3 \times 3 \mathrm{~mm}^{2}, 25 \mathrm{~cm}-$ long. Each fiber was covered with a white acrylic paint as the internal layer and a black aerosol paint as an external one for the light insulation and had two MAPMTs H9500 [8] mounted to the both sides of the bundle. Optical grease was used to improve the light transmission.

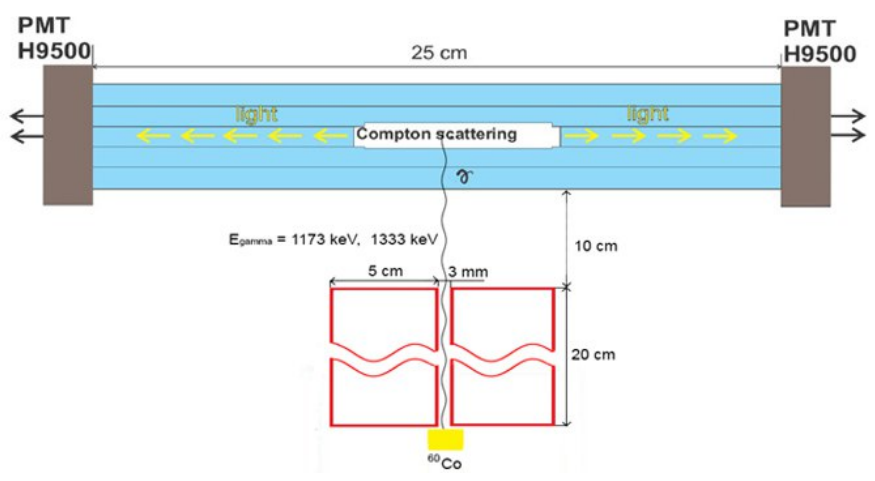

Figure 1: A scheme of the measurements with the NeuRad prototype. The prototype was irradiated by a collimated $\gamma$-source ${ }^{60} \mathrm{Co}$. The gamma rays were focused at the geometrical center of the prototype and signals were collected by a couple of H9500 MAPMTs.

This prototype was irradiated by a collimated ${ }^{60} \mathrm{Co}$ gamma-source with the energies $E_{\gamma}^{(1)}=1173 \mathrm{keV}$ and $E_{\gamma}^{(2)}=1333 \mathrm{keV}$ as showed in the Fig. 1. Signals obtained from the MAPMTs were collected by the DRS4 evaluation board [9] (12 bit at $5 \mathrm{GS} / \mathrm{s})$ and saved for the each detected event. Two channels of the board were employed for collecting signals from the both sides of one fiber. The channel from one side was used for triggering with the threshold of $20 \mathrm{mV}$. On the other side of the fiber, two remaining channels of the board were connected to the neighboring fibers.

\section{Simulations and data processing}

The simulation and data processing were performed within the EXPERTroot framework. A particle transport through the setup was performed using the GEANT4 [12] classes called from the EXPERTroot software. Obtained energy deposits were converted into the MAPMT anode pulses. Each pulse was calculated as a sum of single photoelectron pulses taking into account the following parameters and effects: light output of the scintillating fiber; the efficiency of the light trapping into the fiber due to a total internal reflection; the scintillation decay time; the light attenuation along the fiber; the light collection increase near the ends of the fiber; the light losses at the optical interface; the MAPMT quantum efficiency; the single photoelectron 
amplitude spectrum, and the pulse shape; the spread of the photoelectron avalanche transition time through the dynode system; the cross-talk as a probability of the single photoelectron avalanche developing in the neighboring pixel. The simulated pulse shapes had the same format as in the experimental data. Implementation details can be found in [10].

Certain effects were not taken into account: any dependence of the pulse shape on the amplitude; the cross-talk as the charge sharing of a single photoelectron avalanche between two or more anodes; the partial collection of the light after a diffuse scattering at the fiber faces; the electronic noises; the pulse shape distortion in the readout line. Most of the parameters for the simulations were taken from the data-sheets of the fibers and MAPMT. The single photoelectron pulse shape was fitted to the experimental data. The single photoelectron amplitude spectrum was borrowed from [11], where a similar MAPMT H12700 has been studied.

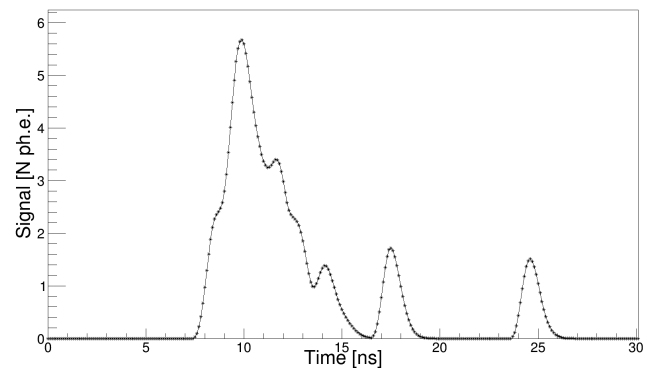

a)

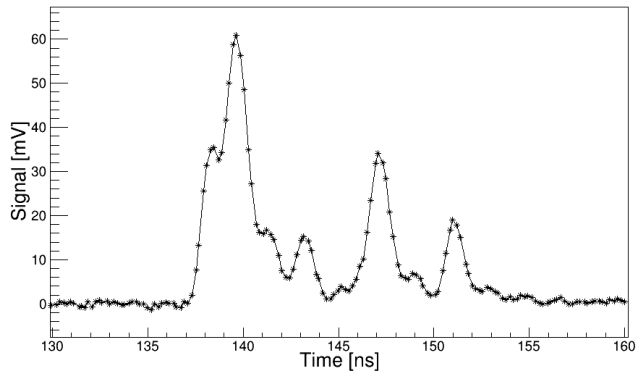

b)

Figure 2: The typical pulse shapes containing about 10 photoelectrons. a) The simulated MAPMT anode output as a result of a composition of several photoelectrons fluctuating according to the assumed distributions b) Typical shape of a measured signal containing about 12 photoelectrons.

Typical pulses obtained in simulations and measurements (shown in the Fig. 2a) and the Fig. 2b) respectively) seem to follow the similar single photoelectron distributions in time and amplitude. However, there is a clear difference between the simulated and the recorded experimental pulses. In the experimental ones one can notice a kind of low-amplitude ringing. It can be due to several reasons, e.g. a stray capacity discharge or influence of the readout line bandwidth, which manifests itself in the damped oscillations caused by the sharp leading edge.

Several standard and rather simple data processing algorithms were implemented into the EXPERTroot framework, such as leading edge discriminator, different event selection filters, alignment and summing up of the pulses, etc. The implemented algorithms allowed us to study the summed pulse and to understand qualitatively its basic features. All algorithms could be applied in the same way to both simulated and experimental data.

Simulated and experimental summed pulses, normalized by their maximum amplitudes, in a logarithmic scale are shown in the Fig. 3. The trigger threshold for the simulated data was set to the 1.7 of the average single photoelectron amplitude. Such a threshold allowed to match the sharp bend at the leading edge (indicated with an arrow in the Fig. 3) in the both pulses. From this picture it can be established that the experimental pulse has the decay profile which includes a fast exponent, faster than the value from the data-sheet, followed by 


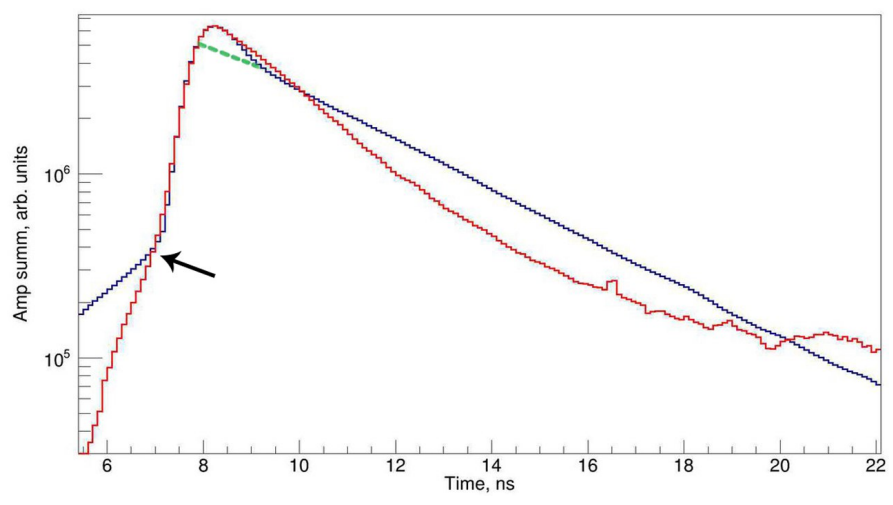

Figure 3: The pulse sums obtained in the measurements (red) and the simulations (blue) in a $\log$ scale.

the long non-exponential tail. For simulated pulse there is a bump before the exponential decay. This feature is getting more prominent if the light losses at the optical interface go down or the cross talks go up. In order to get the quantitative fitting of the parameters and validation of the MC model further measurements are needed.

The parameters of the readout chain needs to be precisely measured using a fast laser. The actual single photoelectron pulse shapes and spectrum should be acquired. Bigger number of pixels should be read out at the same time in order to study all the effects more carefully. As the next step, multichannel electronics based on the TOFPET ASIC [13] will be used for the readout of the NeuRad prototype. A transfer function of these electronics will be implemented into the EXPERTroot software in other to compare the simulated and the experimental data.

\section{Conclusion}

The first small-size prototype of the NeuRad detector was investigated using the $\gamma$-source. The pulse shapes have been obtained using the DRS4 waveform digitizer board and have been studied individually and in average. Processing algorithms were implemented into the EXPERTroot software package. In order to understand the pulse formation mechanism, the measurements were simulated using the EXPERTroot.

The influence of such parameters of the detector as light collection efficiency, interfiber cross talk, detection threshold and luminescence kinetics on the signal shape are understood. For a better understanding of the timing properties, quantitative validation of the MC simulations and complete feasibility study it is necessary to conduct new measurements with the NeuRad prototype recording bigger number of channels and using well calibrated electronics. The results of this study will allow to choose the optimal readout electronics for the entire system.

\section{Acknowledgement}

The authors are grateful to I G Mukha for the stimulating interest and numerous useful advices. This work was partly supported by the Helmholtz Association under grant agreement 
IK-RU-002, RSF 17-12-01367 grant and the MEYS Projects (Czech Republic) LTT17003 and LM2015049.

\section{References}

[1] L.Grigorenko et al., "Studies of light exotic nuclei in vicinity of neutron and proton drip-lines at FLNR JINR" Phys. Usp. 59 321-366 (2016); DOI: 10.3367/UFNe.0186.201604a.0337

[2] M. Winkler et al., The status of the Super-FRS in-flight facility at FAIR, Nucl. Instr. and Meth. in Phys. Res. B 266 (2008) 4183.

[3] Geissel, H., Kiselev, O., Mukha, I., et al., "Expert (exotic Particle Emission and Radioactivity by Tracking) Studies at the Super-FRS Spectrometer", 2015, Exotic Nuclei: EXON-2014 - Proceedings of International Symposium.

[4] Technical Design Report of the EXPERT setup of the Super-FRS Experiment Collaboration, https;//edms.cern.ch/document/1865700

[5] http://er.jinr.ru/

[6] D. Kostyleva et al., GSI Scientific Report 2016171 (2017), DOI:10.15120/GR-2017-1

[7] https://www.crystals.saint-gobain.com/

[8] www.hamamatsu.com/

[9] https://www.psi.ch/drs/

[10] http://er.jinr.ru/neurad.html

[11] J. Adamczewski-Musch et al., Inst. and Exp. Tech. (3) 2018

[12] Nuclear Instruments and Methods in Physics Research A 506 (2003) 250-303, and IEEE Transactions on Nuclear Science 53 No. 1 (2006) 270-278.

[13] http://www.petsyselectronics.com/web/ 